Western University Scholarship@Western

$5-23-2015$

\title{
Ventilation Heterogeneity in Ex-smokers without Airflow Limitation.
}

Damien Pike

Miranda Kirby

Fumin Guo

David G McCormack

Grace Parraga

Follow this and additional works at: https://ir.lib.uwo.ca/biophysicspub

Part of the Medical Biophysics Commons

Citation of this paper:

Pike, Damien; Kirby, Miranda; Guo, Fumin; McCormack, David G; and Parraga, Grace, "Ventilation Heterogeneity in Ex-smokers without Airflow Limitation." (2015). Medical Biophysics Publications. 81.

https://ir.lib.uwo.ca/biophysicspub/81 


\title{
Original Investigations
}

\section{Ventilation Heterogeneity in Ex-smokers without Airflow Limitation}

\author{
Damien Pike, $\mathrm{BSc}^{1}$, Miranda Kirby, $\mathrm{PhD}^{2}$, Fumin Guo, MEng ${ }^{1}$, David G. McCormack, MD, FRCPC ${ }^{3}$, \\ Grace Parraga, $\mathrm{PhD}^{1}$
}

\begin{abstract}
Rationale and Objectives: Hyperpolarized ${ }^{3} \mathrm{He}$ magnetic resonance imaging (MRI) ventilation abnormalities are visible in ex-smokers without airflow limitation, but the clinical relevance of this is not well-understood. Our objective was to phenotype healthy ex-smokers with normal and abnormally elevated ventilation defect percent (VDP).

Materials and Methods: Sixty ex-smokers without airflow limitation provided written informed consent to ${ }^{3} \mathrm{He}$ MRI, computed tomography (CT), and pulmonary function tests in a single visit. ${ }^{3} \mathrm{He}$ MRI VDP and apparent diffusion coefficients (ADCs) were measured for wholelung and each lung lobe as were CT measurements of emphysema (relative area $[R A]$ with attenuation $\leq-950 \mathrm{HU}$, RA ${ }_{950}$ ) and airway morphology (wall area percent [WA\%], lumen area [LA] and LA normalized to body surface area [LA/BSA]).
\end{abstract}

Results: In 42 ex-smokers, there was abnormally elevated VDP and no significant differences for pulmonary function, RA ${ }_{950}$, or airway measurements compared to 18 ex-smokers with normal VDP. Ex-smokers with abnormally elevated VDP reported significantly greater ${ }^{3} \mathrm{He}$ ADC in the apical lung (right upper lobe [RUL], $P=.02$; right middle lobe [RML], $P=.04$; and left upper lobe [LUL], $P=.009$ ). Whole lung $(r=0.40, P=.001)$ and lobar VDP (RUL, $r=0.32, P=.01 ; R M L, r=0.46, P=.002$; right lower lobe $[R L L], r=0.38, P=.003 ; L U L, r=0.35$, $P=.006$; and left lower lobe, $r=0.37, P=.004$ ) correlated with regional ${ }^{3} \mathrm{He}$ ADC. Although whole-lung VDP and CT airway morphology measurements were not correlated, regional VDP was correlated with RUL LA $(r=-0.37, P=.004)$, LA/BSA $(r=-0.42, P=.0008), R L L$ WA $\%(r=0.28, P=.03)$, LA $(r=-0.28, P=.03)$, and LA/BSA $(r=-0.37, P=.004)$.

Conclusions: Abnormally elevated VDP in ex-smokers without airflow limitation was coincident with very mild emphysema detected using $\mathrm{MRI}$ and regional airway remodeling detected using CT representing a subclinical obstructive lung disease phenotype.

Key Words: Hyperpolarized ${ }^{3} \mathrm{He}$ magnetic resonance imaging; computed tomography; airways disease; emphysema.

(c)AUR, 2015

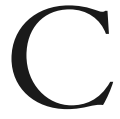
hronic obstructive pulmonary disease (COPD) is characterized by irreversible airflow limitation caused by small airway remodeling, airway obliteration (1), and emphysematous tissue destruction (2). COPD is typically diagnosed after respiratory symptoms become obvious and intolerable (3), and this usually occurs when

\footnotetext{
Acad Radiol 2015; $\mathbf{a}: 1-11$

${ }^{1}$ Current Address: Robarts Research Institute, 1151 Richmond St North, London, Ontario, Canada N6A 5B7.

${ }^{2}$ Current Address: St. Paul's Hospital, Burrard Building, 1081 Burrard St-Room 166, Vancouver, British Columbia, Canada V6Z 1 Y6.

${ }^{3}$ Current Address: London Health Sciences Centre, Victoria Hospital, 800 Commissioners Road East, London, Ontario, Canada N6G 3G4.
}

From the Imaging Research Laboratories, Robarts Research Institute, 1151 Richmond St N, London, ON, Canada N6A 5B7 (D.P., F.G., G.P.); Department of Medical Biophysics, The University of Western Ontario, London, Canada (D.P., G.P.); James Hogg Research Centre, St. Paul's Hospital, University of British Columbia, Vancouver, Canada (M.K.); Graduate Program in Biomedical Engineering (F.G., G.P.); and Division of Respirology, Department of Medicine, The University of Western Ontario, London, Canada (D.G.M.). Received December 18, 2014; accepted April 17, 2015. Address correspondence to: G.P. e-mail: gparraga@robarts.ca

(c) AUR, 2015

http://dx.doi.org/10.1016/j.acra.2015.04.006 spirometry measurements of lung function reflect airflow limitation and obstruction. However, it is well understood that ex- and current-smokers may report normal lung function (3) and mild symptoms, and this may represent an early or "subclinical" phase. A deep understanding of the underlying morphologic changes that accompany this "subclinical" phase is lacking, mainly because methods for evaluating pulmonary function cannot detect very mild or early structure-function abnormalities.

Hyperpolarized ${ }^{3} \mathrm{He}$ magnetic resonance imaging (MRI) ventilation heterogeneity has been shown in patients with pulmonary diseases such as COPD $(4,5)$, asthma $(6,7)$, and cystic fibrosis (8). At the same time, however, preclinical or subclinical ${ }^{3} \mathrm{He}$ ventilation heterogeneity has also been observed in volunteers without clinical signs or symptoms of lung disease such as healthy elderly never-smokers (9), ex-smokers without airflow limitation (10), in second-hand smoke exposed adults (11), and current-smokers without disease (12). To evaluate the underlying anatomic and structural determinants of ventilation heterogeneity, thoracic x-ray computed tomography (CT) has been used to help determine the spatial relationship of airways disease and emphysema with ventilation 
abnormalities. For example, recent work (13) provided evidence that in COPD, ${ }^{3} \mathrm{He}$ ventilation defects represent regions of emphysema and airways disease and that this relationship depends on disease severity. In asthma (7), ${ }^{3} \mathrm{He}$ ventilation heterogeneity was also shown to be spatially related to abnormally remodeled airways. However, the pathophysiological origins and contributions of very mild or subclinical airways disease and emphysema to ventilation heterogeneity in exsmokers without airflow limitation have not been investigated and remain poorly understood.

To better understand the pathophysiological features of ventilation heterogeneity in the "subclinical phase" of obstructive lung disease in otherwise normal healthy exsmokers, we evaluated a group of well-characterized exsmokers without airflow limitation using both MRI and CT. Because of previous research that has suggested that microstructural alveolar remodeling and small airway obliteration occur in subclinical and mild COPD $(1,2,11,12)$, we hypothesized that in normal ex-smokers, ${ }^{3} \mathrm{He}$ ventilation abnormalities would be spatially and quantitatively related to a combination of very mild airways disease and emphysema.

\section{MATERIALS AND METHODS}

\section{Study Participants}

Study participants provided written informed consent to a protocol approved by a local research ethics board and Health Canada, and the protocol was compliant with the Personal Information Protection and Electronic Documents Act (Canada) and Health Insurance Portability and Accountability Act (USA). Research volunteers were recruited from a tertiary health care practice. Each visit was completed in approximately 2 hours when spirometry, plethysmography, the six-minute walk test, St. George's Respiratory Questionnaire, ${ }^{3} \mathrm{He} \mathrm{MRI}$, and CT were completed.

\section{Spirometry and Plethysmography}

Spirometry was performed according to the American Thoracic Society guidelines (14). Whole-body plethysmography was used to measure lung volumes (MedGraphics Corporation, St. Paul, MN), and the attached gas analyzer was used to measure diffusing capacity of the lung for carbon monoxide.

\section{Imaging}

MRI was performed on a 3T Discovery MR750 (General Electric Health Care, Milwaukee, WI) system with subjects in inspiratory breath hold at functional residual capacity $($ FRC $)+1$ L. ${ }^{1} \mathrm{H}$ MRI was acquired before ${ }^{3} \mathrm{He}$ MRI after inhalation of $1 \mathrm{~L}$ high purity, medical grade nitrogen $\left(\mathrm{N}_{2}\right)$ from FRC using the whole-body radiofrequency coil and a fast spoiled gradient-recalled-echo sequence (FGRE; total scan time $\sim 12 \mathrm{~s}$, repetition time $[\mathrm{TR}]=4.3 \mathrm{~ms}$, echo time $[\mathrm{TE}]=1.0 \mathrm{~ms}$, flip angle $=30^{\circ}$, partial echo percent $=62.5 \%$, bandwidth $[\mathrm{BW}]=62.5 \mathrm{kHz}$, field of view $[\mathrm{FOV}]=40 \times 40 \mathrm{~cm}$, matrix size $=128 \times 80$ [zero padded to $128 \times 128$ ], number of excitations [NEXs] $=1$, slice thickness $=15 \mathrm{~mm}$, number of slices $\sim 14$ [depending on subject size], 0 gap). ${ }^{3} \mathrm{He}$ MRI was acquired using a rigid elliptical transmit-receive chest coil (RAPID, Biomedical, Rimpar, Wuerzburg, Germany) with subjects in inspiratory breath-hold after inhalation from FRC of a $1 \mathrm{~L}$ mixture of hyperpolarized ${ }^{3} \mathrm{He}(5 \mathrm{~mL} / \mathrm{kg}$ body weight $)$ diluted with $\mathrm{N}_{2}$. ${ }^{3} \mathrm{He}$ static-ventilation images were acquired using a partial-echo FGRE sequence (total scan time $\sim 10 \mathrm{~s}$, $\mathrm{TR}=3.8 \mathrm{~ms}, \mathrm{TE}=1.0 \mathrm{~ms}$, flip angle $=7^{\circ}$, partial echo percent $=62.5 \%, \mathrm{BW}=62.5 \mathrm{kHz}, \mathrm{FOV}=40 \times 40 \mathrm{~cm}$, matrix size $=128 \times 80$ [zero padded to $128 \times 128$ ], $\mathrm{NEX}=1$, slice thickness $=15 \mathrm{~mm}$, number of slices $\sim 14$ (depending on subject size), 0 gap). ${ }^{3} \mathrm{He}$ diffusion-weighted MR imaging was completed using a centric $\mathrm{k}$-space sampled FGRE sequence (total scan time $\sim 14 \mathrm{~s}, \mathrm{TR}=6.8 \mathrm{~ms}$, $\mathrm{TE}=4.5 \mathrm{~ms}$, flip angle $=8^{\circ}$, partial echo percent $=62.5 \%$, $\mathrm{BW}=62.5 \mathrm{kHz}, \mathrm{FOV}=40 \times 40 \mathrm{~cm}$, matrix size $=128 \times 80$ [zero padded to $128 \times 128$ ], $\mathrm{NEX}=1$, slice thickness $=30 \mathrm{~mm}$, number of slices $\sim 7,0$ gap) that acquired two interleaved slices with and without diffusion sensitization $\left(\mathrm{b}=1.6 \mathrm{~s} / \mathrm{cm}^{2}\right.$, maximum gradient amplitude $(\mathrm{G})=1.94 \mathrm{G} / \mathrm{cm}$, rise $/$ fall time $=0.5 \mathrm{~ms}$, gradient duration $=0.46 \mathrm{~ms}$, and diffusion time $=1.46 \mathrm{~ms}$ ).

CTwas acquired within 30 minutes of MRI using a 64 slice Lightspeed VCT system (General Electric Health Care). Subjects were transported to the CT suite by wheelchair to prevent the potential for exercise-induced changes between MRI and CT image acquisitions. CT was acquired during inspiratory breath-hold of FRC $+1 \mathrm{~L}$ of $\mathrm{N}_{2}$ using a single spiral acquisition from apex to base with subjects in the supine position (detector configuration $=64 \times 0.625 \mathrm{~mm}$, tube voltage $=120 \mathrm{kVp}$, tube current $=100 \mathrm{mAs}$, tube rotation time $=500 \mathrm{~ms}$, and pitch $=1$ ). The total effective dose was $1.8 \mathrm{mSv}$ as calculated using manufacturer settings and the ImPACT CT dosimetry calculator based on Health Protection Agency (UK) NRPB-SR250.

\section{Image Analysis}

Ventilation heterogeneity or regions of "signal void" were quantified as ${ }^{3} \mathrm{He}$ ventilation defect percent (VDP) using semiautomated software generated in MATLAB (Mathworks, Natick, MA) as previously described (15). Lobar VDP was generated by registering the segmented thoracic CT lobe mask from VIDA Pulmonary Workstation 2.0 (VIDA Diagnostics Inc., Coralville, IA) to ${ }^{3} \mathrm{He} \mathrm{MRI}$ ventilation images using deformable registration, and generating VDP for each lobe (right upper lobe [RUL], right middle lobe [RML], right lower lobe [RLL], left upper lobe [LUL], left lower lobe [LLL]) using hierarchical k-means clustering (15).

All ex-smokers were classified as having normal or abnormally elevated VDP using a threshold based on the upper limit 
TABLE 1. Demographic, Pulmonary Function, and Imaging Data

\begin{tabular}{|c|c|c|c|c|}
\hline Parameter $( \pm \mathrm{SD})$ & All $n=60$ & Normal VDP $(n=18)$ & Elevated VDP $(n=42)$ & $P$ Value* \\
\hline Age, y & $69(9)$ & $67(10)$ & $70(9)$ & $.23(1.0)$ \\
\hline Male, $n(\%)$ & $38(63)$ & $9(50)$ & $29(69)$ & $.17(1.0)$ \\
\hline Pack years & $28(16)$ & 27 (14) & $28(17)$ & $.86(1.0)$ \\
\hline Years quit smoking & $19(15)$ & $13(12)$ & $21(15)$ & $.06(1.0)$ \\
\hline $\mathrm{BMI}, \mathrm{kg} / \mathrm{m}^{2}$ & $29(4)$ & $29(5)$ & $30(4)$ & $.5(1.0)$ \\
\hline FVC, $\%_{\text {pred }}$ & 97 (13) & $100(10)$ & $95(14)$ & $.18(1.0)$ \\
\hline $\mathrm{FEV}_{1}, \%_{\text {pred }}$ & $104(13)$ & $106(12)$ & $102(14)$ & $.28(1.0)$ \\
\hline $\mathrm{FEV}_{1} / \mathrm{FVC}$ & $80(6)$ & $80(6)$ & $80(6)$ & $.78(1.0)$ \\
\hline RV/TLC, $\%_{\text {pred }}$ & $103(15)$ & $104(12)$ & $103(16)$ & $.76(1.0)$ \\
\hline $\mathrm{DL}_{\mathrm{co}}, \%_{\text {pred }}$ & $80(20)$ & 87 (16) & 77 (22) & $.11(1.0)$ \\
\hline 6MWD, m & $404(95)$ & $433(81)$ & $392(98)$ & $.12(1.0)$ \\
\hline SGRQ total & $36(26)$ & $22(20)$ & $23(22)$ & $.78(1.0)$ \\
\hline WA $\%$ & $65(2)$ & $65(2)$ & $65(2)$ & $.89(0.89)$ \\
\hline $\mathrm{LA}, \mathrm{mm}^{2}$ & $14(3)$ & $13(2)$ & $14(4)$ & $.36(1.0)$ \\
\hline LA/BSA, $\mathrm{mm}^{2} / \mathrm{m}^{2}$ & $7(2)$ & $7(1)$ & $7(2)$ & $.81(1.0)$ \\
\hline Airway count & $115(37)$ & $106(28)$ & $119(40)$ & $.20(1.0)$ \\
\hline $\mathrm{RA}_{950}, \%$ & $1.2(1.0)$ & $0.8(0.5)$ & $1.4(1.1)$ & $.08(1.0)$ \\
\hline VDP, $\%$ & $6(3)$ & $3(1)$ & 7 (3) & $<.0001(<.0001)$ \\
\hline $\mathrm{ADC}, \mathrm{cm}^{2} / \mathrm{s}$ & $0.28(0.04)$ & $0.26(0.03)$ & $0.29(0.03)$ & $.01(.18)$ \\
\hline
\end{tabular}

ADC, apparent diffusion coefficient; BMI, body mass index; BSA, body surface area; $\mathrm{LL}_{\mathrm{Co}}$, diffusing capacity for carbon monoxide; FEV $\mathrm{V}_{1}$, forced expiratory volume in 1 second; FVC, forced vital capacity; LA, mean fifth generation airway lumen area; 6MWD, six-minute walk distance; $\mathrm{RA}_{950}$, relative area of the lung parenchyma with attenuation $\leq-950 \mathrm{HU}$; RV, residual volume; SD, standard deviation; SGRQ, St. Georges Respiratory Questionnaire; TLC, total lung capacity; VDP, ventilation defect percent; WA\%, mean fifth generation airway wall area percent.

${ }^{*} \mathrm{Holm}$-Bonferroni corrected $P$ Values in parentheses, $P$ Value considered significant when $P<.05$.

of normal $(95 \%$ confidence interval $[\mathrm{CI}])$ of VDP in 51 healthy elderly never-smokers (9) which was calculated using the following equation:

$$
95 \% \mathrm{CL}=\bar{x}+1.96 \cdot \frac{\sigma}{\sqrt{\mathrm{n}}}
$$

where $\bar{x}$ is the mean VDP of the 51 healthy never-smokers, $\sigma$ is the standard deviation of VDP of the 51 healthy neversmokers, and $n$ is the total number of healthy neversmokers. The upper limit of normal (95\% CI) for VDP was calculated to be $4.3 \%$. Therefore, ex-smokers with VDP $<4.3 \%$ were classified as normal, whereas ex-smokers with VDP $\geq 4.3 \%$ were classified as having abnormally elevated VDP.

${ }^{3} \mathrm{He}$ MRI apparent diffusion coefficients (ADCs) were generated as previously described (16).

CT volumes were evaluated using Pulmonary Workstation 2.0 to generate airway wall area percent (WA\%), lumen area (LA), and airway count. It was previously shown that airway LA is related to body surface area (BSA) $(17,18)$, and therefore, LA was normalized to BSA (LA/BSA). CT WA $\%$, LA, and LA/BSA were measured for subsegmental bronchi including RB1, RB5, RB8, LB1, and LB8 airways because each of these feed individual lobes (RB1-RUL, RB5-RML, RB8-RLL, LB1-LUL, and LB8-LLL), and they were measurable for each subject. Emphysema was also measured using Pulmonary Workstation 2.0 including the relative area of the lung with attenuation $\leq-950$ Hounsfield units $(\mathrm{HU})\left(\mathrm{RA}_{950}\right)$ for whole-lung and each individual lung lobe.

\section{Statistics}

All statistical tests were performed in IBM SPSS V22 (SPSS Inc., Chicago, IL). Normality of data was tested using the Shapiro-Wilk test. A one-way analysis of variance was used to compare multiple parameters, and univariate comparisons were investigated using unpaired two-tailed $t$ tests for normally distributed data and Mann-Whitney $U$ tests for non-normally distributed data. Spearman correlations were performed for non-normally distributed data.

\section{RESULTS}

A summary of demographic, pulmonary function test, and imaging measurements is provided in Table 1 for all 60 subjects, whereas Supplemental Table 1 (online) provides a subject listing of data. All participants reported normal spirometry measurements (forced expiratory volume in $1 \mathrm{sec}-$ ond $\left[\mathrm{FEV}_{1}\right]=104 \pm 13 \%, \mathrm{FEV}_{1} /$ forced vital capacity $[\mathrm{FVC}]=80 \pm 6 \%)$ and no subjects reported Global initiative for Chronic Obstructive Lung Disease unclassified COPD (19). Eighteen subjects $(18$ of $60=30 \%$ ) reported normal VDP and $42(42$ of $60=70 \%)$ subjects reported abnormally elevated VDP. As shown in Figure 1 for three representative subjects, volunteers with normal VDP showed small or no ventilation defects along the periphery of the lung, whereas 


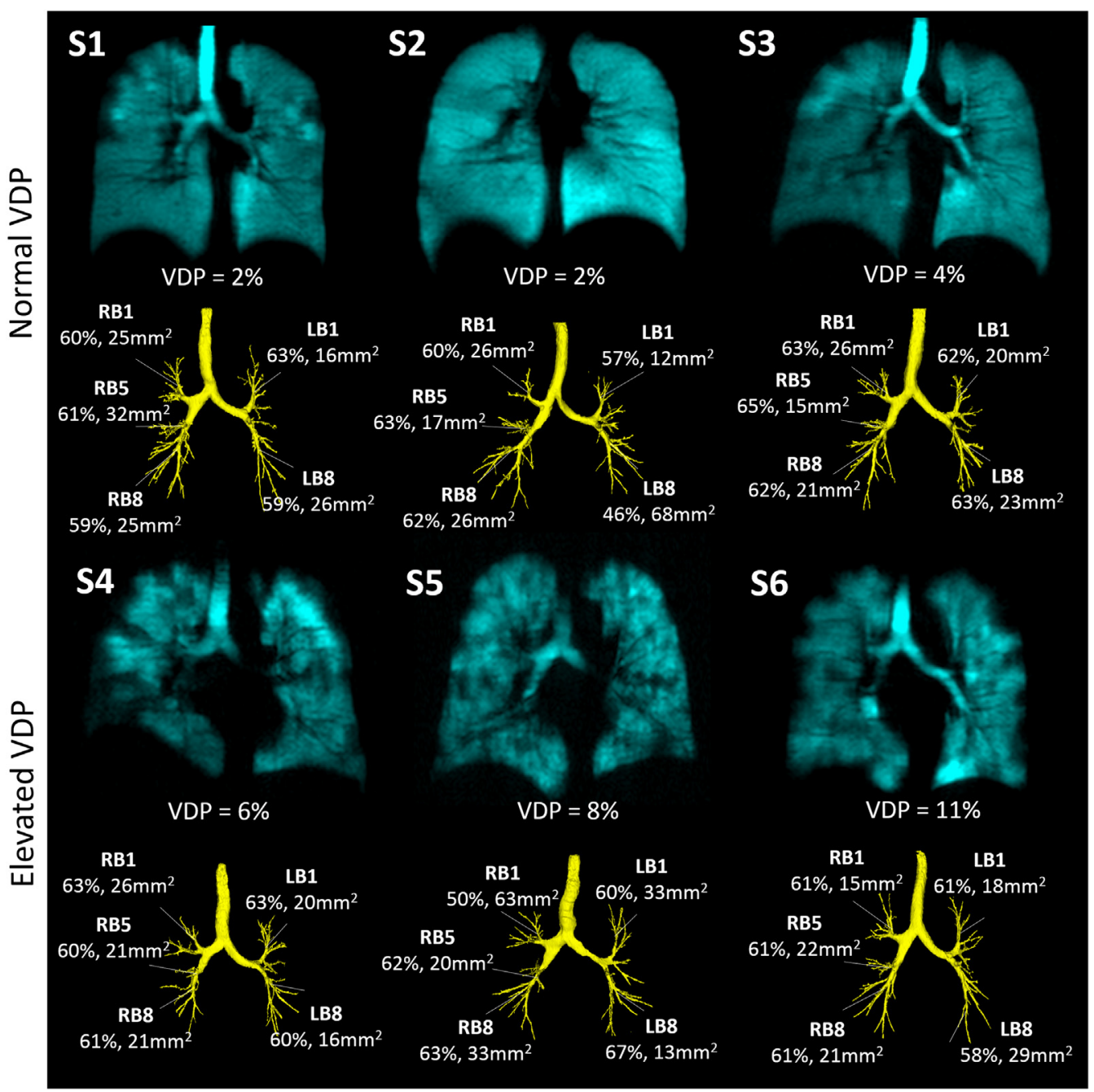

Figure 1. ${ }^{3} \mathrm{He}$ magnetic resonance imaging ventilation and computed tomography airway trees in representative ex-smokers with normal (S1S3) and abnormally elevated ventilation defect percent (VDP) (S4-S6) (airway measurements displayed as wall area percent and lumen area $\left.\left[\mathrm{mm}^{2}\right]\right) \mathrm{S} 1=70$-year-old woman, 12 pack years, forced expiratory volume in 1 second (FEV1) = 93\% and VDP = 2\%; S2 =59-year-old woman, 18 pack years, FEV1 = 97\% and VDP = 2\%; S3 = 51-year-old woman, 20 pack years, FEV1 = 103\% and VDP = 4\%; S4 = 74-year-old man, 50 pack years, FEV1 $=89 \%$ and VDP $=8 \%$; S5 $=79$-year-old man, 10 pack years, FEV1 $=88 \%$ and VDP $=11 \%$; and S6 $=74-$-year-old man, 60 pack years, $\mathrm{FEV} 1=95 \%$ and $\mathrm{VDP}=6 \%$.

participants with abnormally elevated VDP showed evidence of patchy ventilation throughout the lung and on the periphery. The qualitative spatial relationship for patchy ventilation and subsegmental airway morphology reconstructed from CT is also shown in Figure 1.

As shown in Table 1, subjects with abnormally elevated VDP were not significantly different $(P>.05)$ with respect to pulmonary function test or CT measurements, but they did report significantly greater $(P=.01){ }^{3} \mathrm{He}$ ADC than subjects with normal VDP.

Table 2 shows quantitative airway morphology and emphysema measurements for subjects with normal and abnormally elevated VDP. No significant differences were observed for whole-lung airway (mean of fifth generation airways) WA $\%$ $(P=.88)$, LA $(P=.50)$, or LA/BSA $(P=.57)$, and this was consistent in all lung lobes. Table 2 and Figure 2 show that only ${ }^{3} \mathrm{He}$ MRI ADC was significantly greater in the apical lung (RUL, $P=.02$; RML, $P=.04$; and LUL, $P=.009$ ) in subjects with elevated VDP.

Table 3 shows the relationships for VDP with airway morphology and emphysema measurements. Whole lung VDP was significantly correlated with whole lung ADC ( $\mathrm{r}=0.40, P=.001)$ and whole lung $\mathrm{RA}_{950}(\mathrm{r}=0.34$, $P=.008)$. As shown in Table 3 and in more detail in Figure 3, regional VDP correlated with regional ADC in each of the lung lobes (RUL: $\mathrm{r}=0.32, P=.01$; RML: $\mathrm{r}=0.46, P=.002$; RLL: $\mathrm{r}=0.38, P=.003$; LUL: $\mathrm{r}=0.35, P=.006$; and LLL: $\mathrm{r}=0.37, P=.004)$. As shown in Table 3, whole lung VDP did not correlate with whole lung (mean of fifth generation airways) WA\%, LA, or LA/ BSA. There were no significant correlations for regional VDP and airway morphologic measurements in the RML 
TABLE 2. Whole Lung and Regional Measurements for Ex-smokers with Normal and Elevated VDP

\begin{tabular}{|c|c|c|c|}
\hline Parameter $( \pm \mathrm{SD})$ & Normal VDP $(n=18)$ & Elevated VDP $(n=42)$ & $P$ Value \\
\hline \multicolumn{4}{|l|}{ Whole lung } \\
\hline WA\%* & $65(2)$ & $65(2)$ & .88 \\
\hline $\mathrm{LA}, \mathrm{mm}^{2 *}$ & $13(2)$ & $14(4)$ & .50 \\
\hline LA/BSA, $\mathrm{mm}^{2} / \mathrm{m}^{2 *}$ & $7(1)$ & $7(2)$ & .57 \\
\hline VDP, $\%$ & $3(1)$ & $7(3)$ & $<.0001$ \\
\hline $\mathrm{ADC}, \mathrm{cm}^{2} / \mathrm{s}$ & $0.26(0.03)$ & $0.29(0.03)$ & .01 \\
\hline $\mathrm{RA}_{950}, \%$ & $0.8(0.5)$ & $1.4(1.1)$ & .08 \\
\hline \multicolumn{4}{|l|}{ Right upper lobe } \\
\hline RB1 WA\% & $62(2)$ & $61(4)$ & .29 \\
\hline $\mathrm{RB} 1 \mathrm{LA}, \mathrm{mm}^{2}$ & $25(5)$ & $25(11)$ & .64 \\
\hline RB1 LA/BSA, $\mathrm{mm}^{2} / \mathrm{m}^{2}$ & $13(3)$ & $13(6)$ & .39 \\
\hline VDP, $\%$ & $3(2)$ & $5(4)$ & .02 \\
\hline $\mathrm{ADC}, \mathrm{cm}^{2} / \mathrm{s}$ & $0.24(0.03)$ & $0.27(0.03)$ & .02 \\
\hline $\mathrm{RA}_{950}, \%$ & $0.7(0.6)$ & $1.2(1.2)$ & .08 \\
\hline \multicolumn{4}{|l|}{ Right middle lobe } \\
\hline RB5 WA\% & $63(4)$ & $62(4)$ & .25 \\
\hline RB5 LA, mm² & $22(13)$ & $21(8)$ & .80 \\
\hline RB5 LA/BSA, $\mathrm{mm}^{2} / \mathrm{m}^{2}$ & $12(7)$ & $11(4)$ & .99 \\
\hline VDP, $\%$ & $6(9)$ & $9(10)$ & .04 \\
\hline $\mathrm{ADC}, \mathrm{cm}^{2} / \mathrm{s}$ & $0.24(0.03)$ & $0.27(0.04)$ & .04 \\
\hline $\mathrm{RA}_{950}, \%$ & $1.6(1.0)$ & $2.0(1.8)$ & .79 \\
\hline \multicolumn{4}{|l|}{ Right lower lobe } \\
\hline RB8 WA\% & $62(3)$ & $62(3)$ & .43 \\
\hline RB8 LA, $\mathrm{mm}^{2}$ & $21(8)$ & $21(7)$ & .99 \\
\hline RB8 LA/BSA, $\mathrm{mm}^{2} / \mathrm{m}^{2}$ & $11(5)$ & $11(4)$ & .67 \\
\hline VDP, $\%$ & $5(3)$ & $8(6)$ & .02 \\
\hline $\mathrm{ADC}, \mathrm{cm}^{2} / \mathrm{s}$ & $0.25(0.03)$ & $0.27(0.03)$ & .07 \\
\hline $\mathrm{RA}_{950}, \%$ & $0.6(0.4)$ & $1.0(1.0)$ & .42 \\
\hline \multicolumn{4}{|l|}{ Left upper lobe } \\
\hline LB1 WA\% & $63(3)$ & $63(3)$ & .52 \\
\hline LB1 LA, $\mathrm{mm}^{2}$ & $15(4)$ & $18(6)$ & .19 \\
\hline LB1 LA/BSA, $\mathrm{mm}^{2} / \mathrm{m}^{2}$ & $8(2)$ & $9(3)$ & .34 \\
\hline VDP, $\%$ & $4(4)$ & $8(8)$ & .02 \\
\hline $\mathrm{ADC}, \mathrm{cm}^{2} / \mathrm{s}$ & $0.25(0.03)$ & $0.28(0.04)$ & .009 \\
\hline $\mathrm{RA}_{950}, \%$ & $1.0(0.6)$ & $1.8(1.7)$ & .22 \\
\hline \multicolumn{4}{|l|}{ Left lower lobe } \\
\hline LB8 WA\% & $59(4)$ & $60(4)$ & .68 \\
\hline LB8 LA, $\mathrm{mm}^{2}$ & $30(11)$ & $28(12)$ & .27 \\
\hline LB8 LA/BSA, $\mathrm{mm}^{2} / \mathrm{m}^{2}$ & $17(8)$ & $14(6)$ & .13 \\
\hline VDP, \% & $3(1)$ & $7(3)$ & $<.0001$ \\
\hline $\mathrm{ADC}, \mathrm{cm}^{2} / \mathrm{s}$ & $0.25(0.03)$ & $0.27(0.04)$ & .07 \\
\hline $\mathrm{RA}_{950}, \%$ & $0.7(0.4)$ & $1.1(1.1)$ & .42 \\
\hline
\end{tabular}

$\mathrm{ADC},{ }^{3} \mathrm{He}$ magnetic resonance imaging apparent diffusion coefficient; BSA, body surface area; LA, lumen area; LB1, left upper lobe apical bronchus; LB8, left lower lobe subsegmental bronchus; RA950, relative area of the lung parenchyma with attenuation $\leq-950 \mathrm{HU}$; RB1, right upper lobe apical bronchus; RB5, right middle lobe lateral bronchus; RB8, right lower lobe subsegmental bronchus; SD, standard deviation; VDP, ${ }^{3} \mathrm{He}$ magnetic resonance imaging ventilation defect percent; WA\%, wall area percent.

*Mean of fifth generation airways.

(RB5 morphology), LUL (LB1 morphology), or LLL (LB8 morphology). However, as shown Figure 4 in more detail, RUL VDP significantly correlated RB1 morphologic measurements (LA: $\mathrm{r}=-0.37, P=.004$ and LA/BSA: $\mathrm{r}=-0.42, P=.0008)$ and RLL VDP significantly correlated with RB8 morphologic measurements (WA\%: $r=0.28$, $P=.03$; LA: $\mathrm{r}=-0.28, P=.03$; and LA/BSA: $\mathrm{r}=-0.37$, $P=.004)$.

\section{DISCUSSION}

We acquired ${ }^{3} \mathrm{He}$ MRI and CT in 60 ex-smokers without airflow limitation and made the following observations: 1) 42 of 60 volunteers reported abnormally elevated ${ }^{3} \mathrm{He}$ VDP, and these subjects reported normal airflow and lung volume measurements that were not significantly different from 18 ex-smokers with normal VDP, 2) there was significantly 

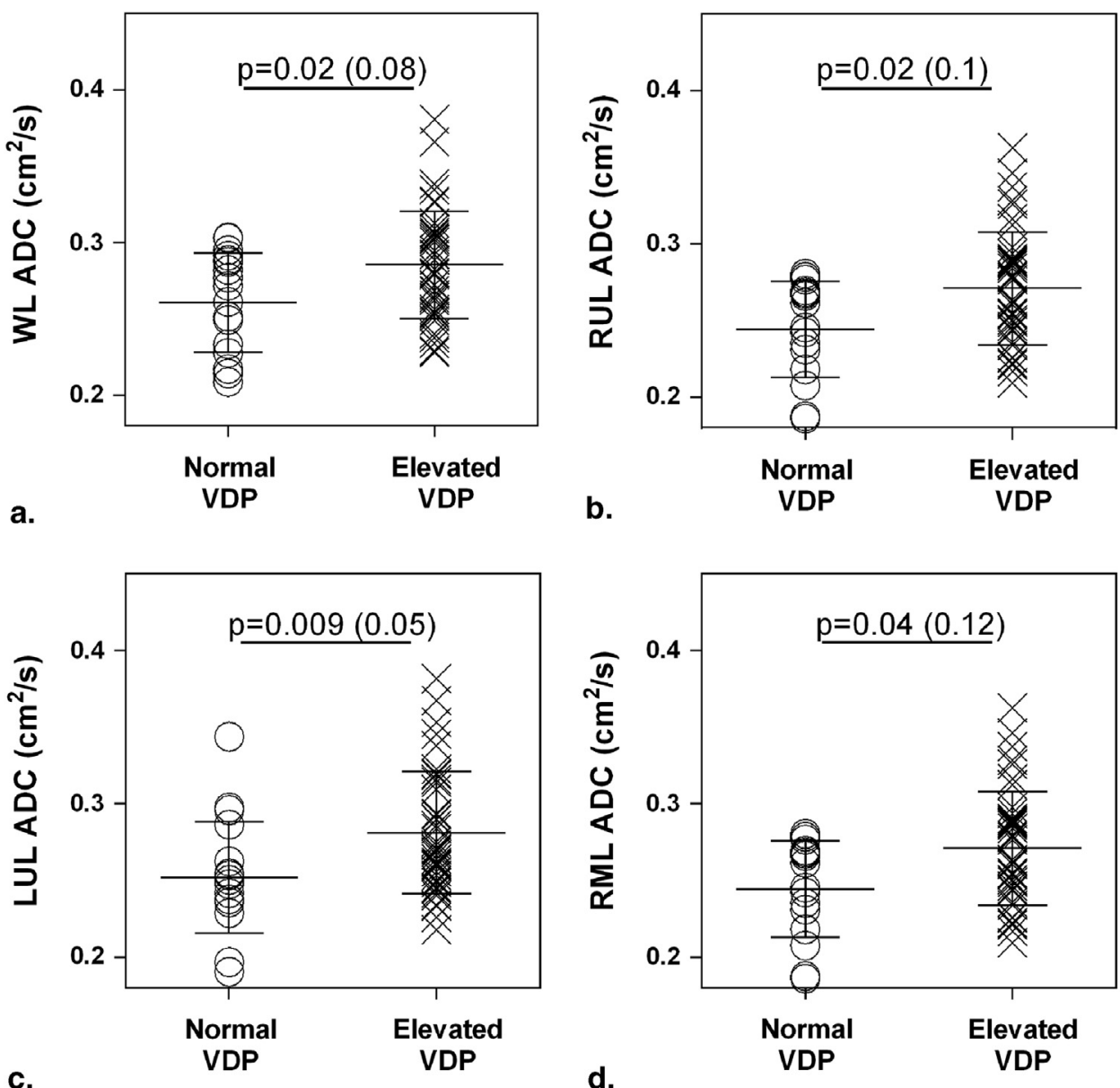

Figure 2. Whole lung (WL) and regional emphysema measurements in ex-smokers with normal ( $n=18$ ) and abnormally elevated ventilation defect percent (VDP; $n=42$; Holm-Bonferroni corrected $P$ Values in parentheses). ADC was significantly greater in apical lung in subjects with elevated VDP. After correcting for multiple comparisons,
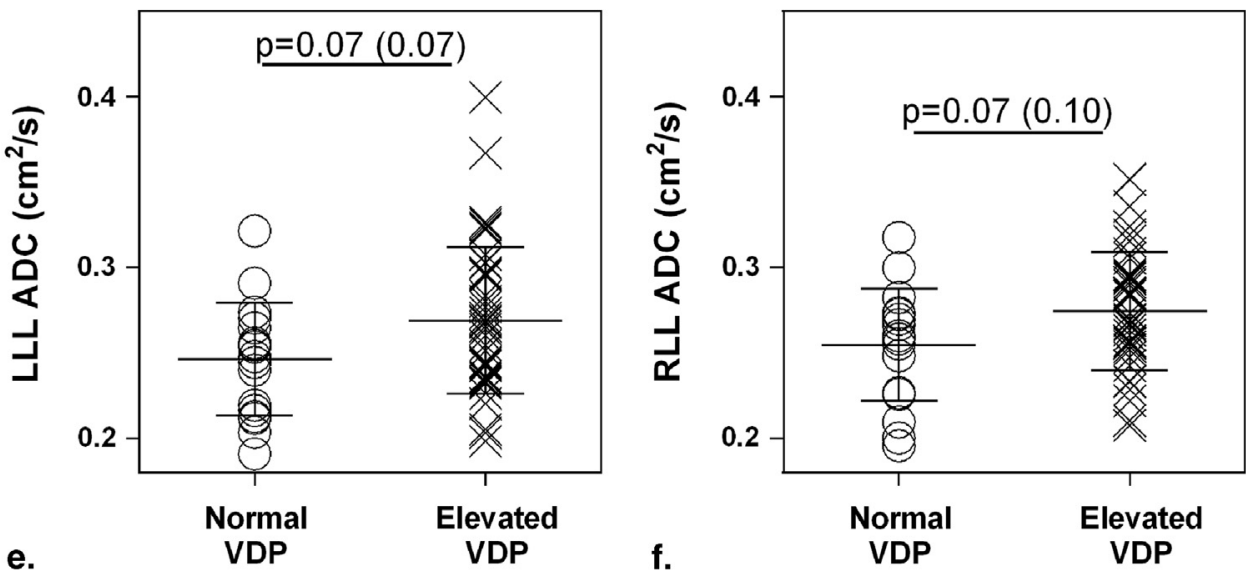
only the comparison of LUL ADC remained on the threshold of significance. (a) Whole lung ADC ( $P=.02$, corrected 0.08), (b) right upper lobe ADC $(P=.02$, corrected 0.1 ), (c) left upper lobe ADC $(P=.009$, corrected 0.05), (d) right middle lobe $\operatorname{ADC}(P=.04$, corrected 0.12), (e) left lower lobe ADC $(P=.07$, corrected 0.07$)$, and (f) right lower lobe ADC $(P=.07$, corrected $0.10)$. ADC, apparent diffusion coefficient; LLL, left lower lobe; LUL, left upper lobe; RLL, right lower lobe; $\mathrm{RML}$, right middle lobe; RUL, right upper lobe.

greater (worse) apical lung ${ }^{3} \mathrm{He}$ ADC in the 42 ex-smokers with abnormally elevated VDP as compared to ex-smokers with normal VDP, but there were no other imaging differences between the subgroups, 3$)^{3} \mathrm{He}$ VDP was significantly correlated with whole lung and regional ADC, and 4) whole lung ${ }^{3} \mathrm{He}$ VDP was not correlated with whole lung airway morphologic measurements, although RUL and RLL VDP was associated with thicker airway walls (WA\%) and narrowed airway lumen (LA) in those regions of interest.
We observed that 42 ex-smokers reported VDP $\geq 95 \%$ CI for VDP in never-smokers (9). Although all participants had a significant smoking history (mean pack years $=28$ ), spirometry and CT measurements showed no evidence of lung function decline or lung destruction $\left(\mathrm{FEV}_{1} /\right.$ $\mathrm{FVC}=80 \pm 6 \%$, mean $\mathrm{FEV}_{1} \%_{\text {pred }}=104 \pm 13 \%$, mean $\left.\mathrm{RA}_{950}=1.2 \pm 1.0 \%\right)$. The cutoffs for diagnosing airflow obstruction using spirometry are well established as $\mathrm{FEV}_{1}$ / FVC $<70 \%$ and/or (unclassified) $\mathrm{FEV}_{1} \%_{\text {pred }}<80 \%$ (3), and 
TABLE 3. Relationship of ${ }^{3} \mathrm{He}$ MRI VDP with Airways Disease and Emphysema Measurements

\begin{tabular}{|c|c|c|}
\hline Parameter & Spearman $r$ & $P$ Value \\
\hline \multicolumn{3}{|l|}{ Whole lung VDP } \\
\hline WA\%* ${ }^{*}$ & 0.002 & .99 \\
\hline $\mathrm{LA}^{*}$ & 0.04 & .76 \\
\hline LA/BSA* & -0.04 & .75 \\
\hline ADC & 0.40 & .001 \\
\hline $\mathrm{RA}_{950}$ & 0.34 & .008 \\
\hline \multicolumn{3}{|c|}{ Right upper lobe VDP } \\
\hline RB1 WA\% & 0.24 & .06 \\
\hline RB1 LA & -0.37 & .004 \\
\hline RB1 LA/BSA & -0.42 & .0008 \\
\hline ADC & 0.32 & .01 \\
\hline $\mathrm{RA}_{950}$ & 0.15 & .27 \\
\hline \multicolumn{3}{|c|}{ Right middle lobe VDP } \\
\hline RB5 WA\% & -0.11 & .40 \\
\hline RB5 LA & -0.003 & .98 \\
\hline RB5 LA/BSA & -0.02 & .91 \\
\hline ADC & 0.46 & .002 \\
\hline $\mathrm{RA}_{950}$ & 0.24 & .07 \\
\hline \multicolumn{3}{|c|}{ Right lower lobe VDP } \\
\hline RB8 WA\% & 0.28 & .03 \\
\hline RB8 LA & -0.28 & .03 \\
\hline RB8 LA/BSA & -0.37 & .004 \\
\hline ADC & 0.38 & .003 \\
\hline $\mathrm{RA}_{950}$ & 0.21 & .11 \\
\hline \multicolumn{3}{|c|}{ Left upper lobe VDP } \\
\hline LB1 WA\% & 0.07 & .62 \\
\hline LB1 LA & 0.13 & .33 \\
\hline LB1 LA/BSA & -0.01 & .94 \\
\hline ADC & 0.35 & .006 \\
\hline $\mathrm{RA}_{950}$ & 0.23 & .08 \\
\hline \multicolumn{3}{|c|}{ Left lower lobe VDP } \\
\hline LB8 WA\% & 0.04 & .73 \\
\hline LB8 LA & 0.05 & .68 \\
\hline LB8 LA/BSA & -0.02 & .86 \\
\hline ADC & 0.37 & .004 \\
\hline $\mathrm{RA}_{950}$ & 0.26 & .05 \\
\hline
\end{tabular}

ADC, ${ }^{3} \mathrm{He} \mathrm{MRI}$ apparent diffusion coefficient; BSA, body surface area; LA, lumen area; LB1, left upper lobe apical bronchus; LB8, left lower lobe subsegmental bronchus; MRI, magnetic resonance imaging; $R A_{950}$, relative area of the lung parenchyma with attenuation $\leq-950 \mathrm{HU}$; RB1, right upper lobe apical bronchus; RB5, right middle lobe lateral bronchus; RB8, right lower lobe subsegmental bronchus; VDP, ${ }^{3} \mathrm{He} \mathrm{MRI}$ ventilation defect percent; WA $\%$, wall area percent.

*Mean of fifth generation airways.

various thresholds ranging from $\mathrm{RA}_{950}=7 \%-10 \%$ have been used to identify emphysema predominant phenotypes (20), and we noted that the spirometry and CT measurements from the ex-smokers in our study did not cross any of these thresholds. At the same time, we were surprised that these same measurements showed no significant differences between subjects with elevated VDP compared to subjects with normal VDP. Previous work (10) in "healthy" smokers showed significantly different MRI ventilation measure- ments as compared to never-smokers and COPD smokers and our findings build on this previous work by showing that ex-smokers with abnormally elevated VDP also showed significantly greater whole-lung and apical lung ${ }^{3} \mathrm{He}$ ADC. In this regard, it is important to note that ${ }^{3} \mathrm{He} A D C$ provides a surrogate measure of microstructural emphysema (21) and was previously shown to be sensitive to very mild emphysematous changes by detecting differences in subjects exposed only to second-hand smoke (11). We observed that subjects with elevated VDP reported greater whole-lung and apical lung ${ }^{3} \mathrm{He}$ ADC. Previous work (22) evaluated the anatomic ADC distribution in more severe COPD and established the presence of elevated ADC in the apical lung regions, and this is in agreement with predominant centrilobular emphysema in such patients. However, to our knowledge, the spatial distribution of ADC in ex-smokers without COPD has not yet been evaluated. We did not observe differences in CT emphysema $\left(\mathrm{RA}_{950}\right)$ measurements. However, previous research (23) showed that CT-derived emphysema measurements may underestimate the extent of emphysema in very mild disease. The mean $\mathrm{RA}_{950}$ in the ex-smokers in our study was very low (mean $\mathrm{RA}_{950}=1.2 \%$ ), and this may reflect why CT $\mathrm{RA}_{950}$ was not significantly different between subgroups. Nevertheless, these results suggest that ex-smokers with a significant smoking history and abnormal ${ }^{3} \mathrm{He}$ VDP but without airflow limitation may also have a mild or subclinical form of centrilobular emphysema that is not detected using CT or conventional pulmonary function tests.

Whole lung and regional VDP were correlated with ADC which suggests that ventilation abnormalities and very mildly abnormal parenchyma coexisted in these otherwise normal ex-smokers. We evaluated regional (lobar) correlations for ${ }^{3} \mathrm{He}$ VDP and ADC because a direct spatial comparison previously described in COPD patients (13) was not feasible here. This previous approach (13) evaluated the direct spatial relationship between ${ }^{3} \mathrm{He}$ ventilation defects and emphysema by evaluating the overlap of $\mathrm{RA}_{950}$ (emphysema) regions and ${ }^{3} \mathrm{He}$ ventilation defects. As compared to COPD patients with severe emphysema $\left(\mathrm{RA}_{950}>15 \%\right)$, the ex-smokers here reported very low or normal $\mathrm{RA}_{950}$ and therefore the spatial overlap of ${ }^{3} \mathrm{He}$ ventilation defects and $\mathrm{RA}_{950}$ could not be evaluated. We wonder about the long-term consequences of these very mild abnormalities in ventilation and emphysema measurements and whether these are predictors of a transition to COPD. Future longitudinal evaluations in these subjects are warranted and will be undertaken.

It was not expected that whole-lung airway measurements would show differences between subgroups because it is widely appreciated that obstructive disease is regionally (and not uniformly) distributed in the lung (24). However, it was surprising that we did not detect or measure abnormal or different regional airway morphologies in the ex-smokers with abnormally elevated VDP, especially because wholelung and lobar VDP was significantly greater in these participants. We think one explanation for this may be related to 


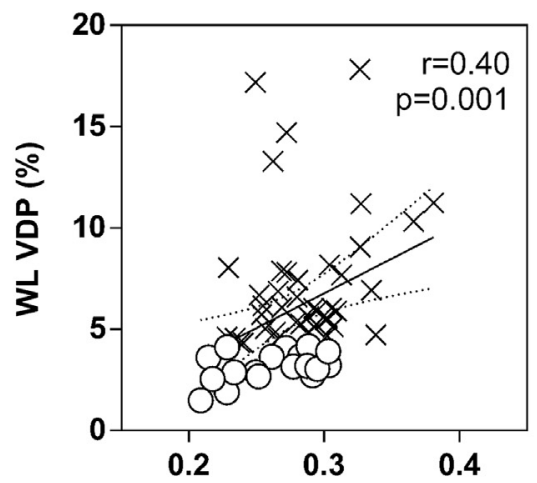

a.

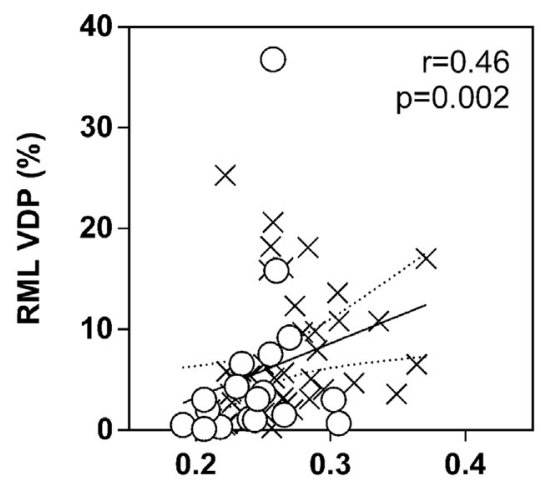

d.

RML ADC $\left(\mathrm{cm}^{2} / \mathrm{s}\right)$
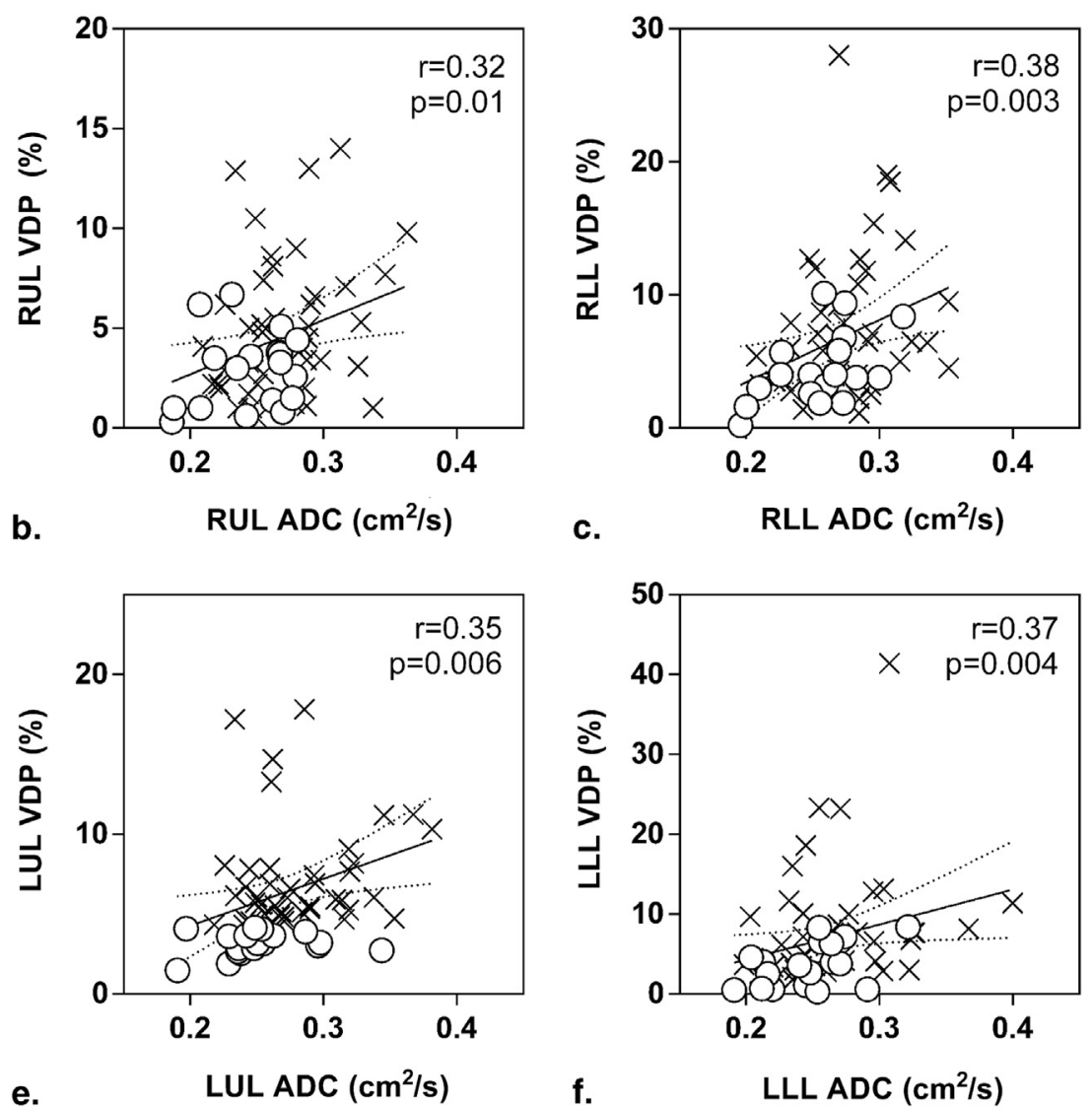

f.

$\times$ Elevated VDP

Figure 3. Relationships for whole lung and regional ventilation defect percent (VDP) with ${ }^{3} \mathrm{He}$ magnetic resonance imaging emphysema measurements. (a) Whole lung (WL) VDP correlated with WL ADC ( $r=0.40, P=.001)$, (b) RUL VDP correlated with RUL ADC ( $r=0.32, P=.01)$, (c) RLL VDP correlated with RLL ADC ( $r=0.38, P=.003)$, (d) RML VDP correlated with RML ADC ( $r=0.46, P=.002)$, (e) LUL VDP correlated with LUL $\operatorname{ADC}(r=0.35, P=.006)$, and (f) LLL VDP correlated with LLL ADC $(r=0.37, P=.004)$. ADC, apparent diffusion coefficient; LLL, left lower lobe; LUL, left upper lobe; RLL, right lower lobe; RML, right middle lobe; RUL, right upper lobe.

the airways we measured and the fact that they may not have been distal enough to reflect such mild disease. We observed significant correlations between airway morphology and VDP in the RUL and RLL. Previous studies $(25,26)$ have shown that RUL and RLL airway morphology is significantly correlated with whole-lung function (assessed by spirometry) in COPD, but these studies did not investigate regional relationships with function. The observation that thickened airway walls and narrowed airway lumens correlate with increasing ventilation heterogeneity in the RUL, and RLL suggests that there may be a structure-function relationship between airway morphology and ventilation in exsmokers. However, we must caution that this was not observed for the left lung lobes. At the same time, we note that left lung airway morphology and its relationship to regional and global lung function has largely been underinvestigated compared to right lung airway morphology, perhaps because the relative sizes of the major airways, make right lung investigations more easily undertaken. These findings may also be highlighting some of the limitations of CT and ad- vantages of MRI for pulmonary imaging and characterization of very mild lung disease. As described elsewhere $(27,28)$, CT measurements are limited because of the inherent spatial resolution limit achievable on thoracic CT scans. On the other hand, MRI ADC provides a way to reveal subtle parenchymal changes to the alveolar microstructure, and the subjects in our study certainly exemplify a patient group in whom MRI is highly advantageous.

Previous work with micro-CT has established that small airway obliteration and not macroscopic emphysema may be the dominant contributor to airflow obstruction in the early and mild COPD grades $(1,2)$. At the same time, ${ }^{3} \mathrm{He}$ MRI studies have provided evidence that mild microstructural alveolar remodeling occurs in current-smokers without COPD $(12,29)$ and individuals with no $(30)$ or very mild exposure to cigarette smoke (11). Although it is not known definitively how mild alveolar abnormalities influence airflow, taken together, these previous findings suggest that small airways disease and mild emphysema are both present in elderly never-, ex-, or current-smokers without COPD. In the 


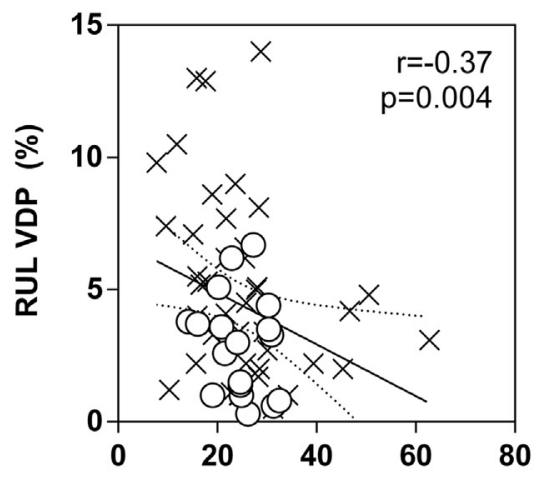

a.

R RB1 LA $\left(\mathrm{mm}^{2}\right)$

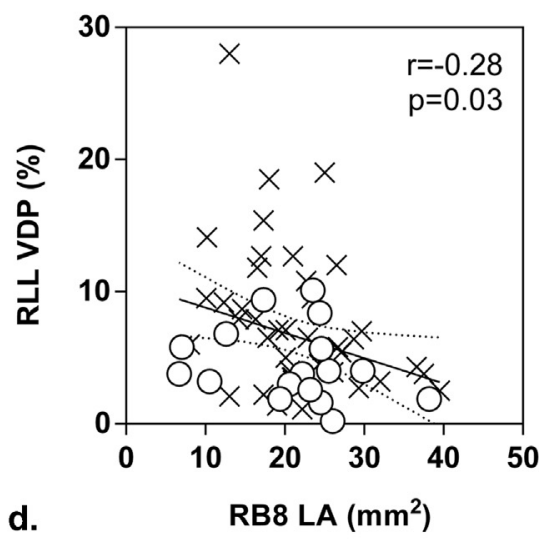

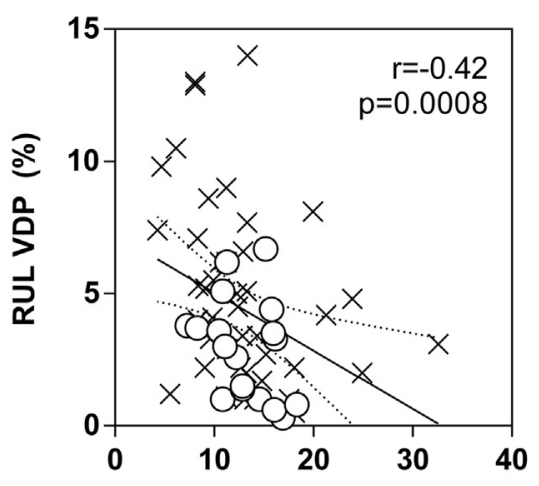

b.

RB1 LA/BSA $\left(\mathrm{m}^{2} / \mathrm{mm}^{2}\right)$

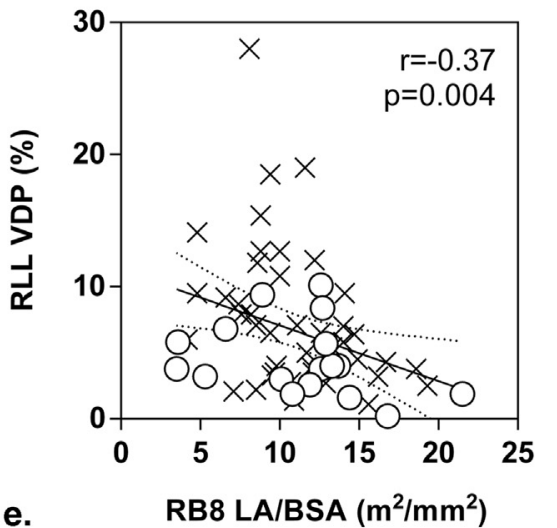

e.

○ Normal VDP

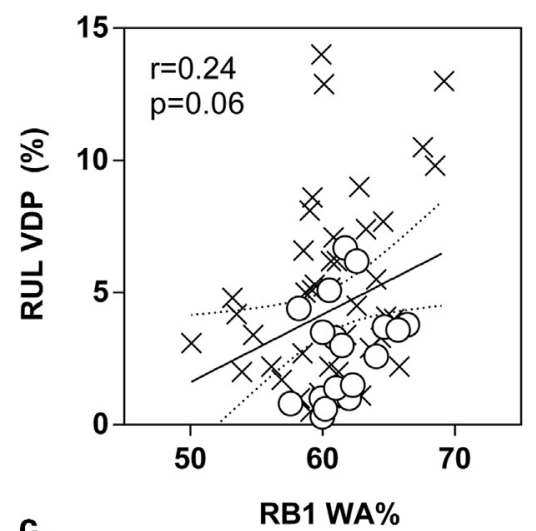

c.

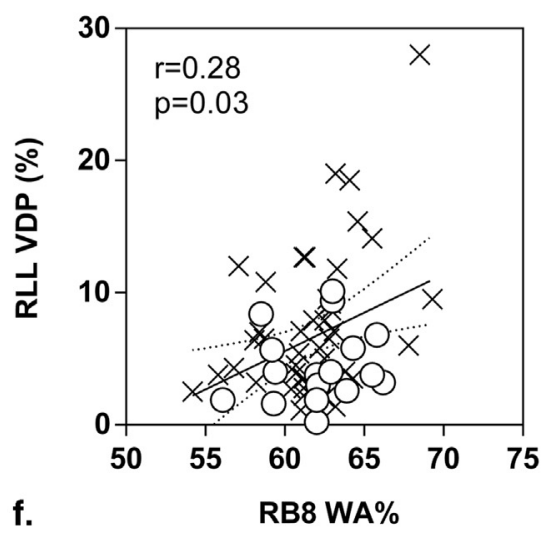

Figure 4. Relationships for regional ventilation defect percent (VDP) with computed tomography airways disease measurements. Right upper lobe (RUL) VDP correlated with (a) RB1 lumen area (LA) $(r=-0.37, P=.004)$, (b) RB1 LA/body surface area $(\mathrm{BSA})(\mathrm{r}=-0.42, P=.0008)$ but not (c) RB1 wall area percent (WA\%) $(r=0.24, P=.06)$. Right lower lobe (RLL) VDP correlated with (d) RB8 LA ( $r=-0.28, P=.03)$, (e) RB8 LA/BSA $(r=-0.37, P=.004)$, and (f) RB8 WA $\%(r=0.28, P=.03)$.

present study, we observed spatial and quantitative relationships for ventilation defects with subsegmental CT airway morphologic abnormalities and ${ }^{3} \mathrm{He}$ ADC. This is in agreement with the hypothesis that ventilation abnormalities and mild emphysema coexist in normal ex-smokers.

We must acknowledge several limitations. We measured five individual subsegmental airways (RB1, RB5, RB8, LB1, and LB8) and not all subsegmental airways. We chose to evaluate these five airways because they were most easily visible and measurable on thoracic CT and directly lead to the individual lung lobes of interest. Although it would be possible to quantitatively evaluate more distal subsegmental airways, the reproducibility of these measurements in all subjects would be difficult to determine. After correcting for familywise error, the comparisons between whole-lung and regional ADC between groups did not remain statistically significant. Although these corrected comparisons did not reach statistical significance, the trend toward elevated VDP and ADC suggests a subclinical centrilobular emphysema phenotype. Although we think the results of our analysis point to very mild emphysema and airways disease in ex-smokers without airflow limitation, we also believe that these findings should be investigated further in a larger ex-smoker population. Our findings were derived on a VDP threshold determined in never-smokers from a previous ${ }^{3} \mathrm{He}$ MRI study (9). The range of VDP for all subjects in our study was $2 \%-$ $18 \%$, and the VDP data distribution was not normally distributed, but reflected a Poisson distribution. This may explain why 42 of 60 subjects reported abnormal VDP. If a greater threshold (vs. 95\% CI) was implemented for abnormal VDP, fewer ex-smokers would have been classified with abnormally elevated VDP. For example, if the abnormal VDP threshold was $5 \%, 33$ of 60 ex-smokers would have been classified with abnormally elevated VDP. Another inherent limitation is that we did not acquire paired inspiratory-expiratory CT scans which prevented us from using recently developed parametric response mapping (PRM) techniques (31) to quantify air trapping in these subjects. Comparisons and spatial correlations between PRM measurements and ${ }^{3} \mathrm{He}$ MRI ventilation and ADC measurements may have provided novel information about the underlying structure-function relationships of preclinical or subclinical forms of COPD which we could not uncover using measurements from inspiratory $\mathrm{CT}$ alone. We must also acknowledge that ${ }^{3} \mathrm{He}$ MRI may 
only be used at a limited number of research centers worldwide because of the limited supply of ${ }^{3} \mathrm{He}$ gas and need for specialized equipment and software. However, pulmonary functional MRI using hyperpolarized ${ }^{129} \mathrm{Xe}$ is emerging as an attractive alternative with the potential for implementation and translation. In this regard, longitudinal measurements in these 60 ex-smokers will be undertaken using ${ }^{129} \mathrm{Xe}$ and more conventional ${ }^{1} \mathrm{H}$ MRI methods. Finally, we also recognize that although this study evaluated 60 ex-smokers, a relatively large study by MRI standards, the sample size may have limited our ability to detect significant relationships with CT airway morphology and emphysema measurements and for detecting significant differences in ADC after familywise error correction. We also acknowledge that we focused only on exsmokers without airflow limitation; therefore, future work investigating MRI ventilation measurements in currentsmokers would provide an understanding of acute effects of cigarette smoke on pulmonary ventilation.

In conclusion, in ex-smokers without airflow limitation, ${ }^{3} \mathrm{He}$ MRI identified a subgroup with abnormal ventilation. Although there were no other pulmonary function or CT differences in ex-smokers with normal or abnormal ventilation, there were spatial relationships between ventilation defects with both elevated ADC and abnormal subsegmental airway morphology. These data suggest that a combination of very mild airways disease and microstructural emphysematous destruction are present in ex-smokers without airflow limitation warranting further longitudinal investigation.

\section{ACKNOWLEDGMENTS}

Funding source: The authors acknowledge funding from a Canadian Institutes of Health Research (CIHR) Team Grant for the Thoracic Imaging Network of Canada, CIHR Operating Grant MOP\# 106437 and the Canadian Respiratory Research Network (CRRN). Dr. Parraga also gratefully acknowledges salary support from a CIHR New Investigator award.

The authors thank Sandra Blamires CCRC, for clinical coordination, Andrew Wheatley BSc, for production and dispensing of ${ }^{3} \mathrm{He}$ gas, and Trevor Szekeres RTMR, for MRI of research volunteers.

\section{SUPPLEMENTARY DATA}

Supplementary data related to this article can be found at http://dx.doi.org/10.1016/j.acra.2015.04.006.

\section{REFERENCES}

1. Hogg JC, Chu F, Utokaparch S, et al. The nature of small-airway obstruction in chronic obstructive pulmonary disease. The New England journal of medicine 2004; 350(26):2645-2653.

2. McDonough JE, Yuan R, Suzuki M, et al. Small-airway obstruction and emphysema in chronic obstructive pulmonary disease. The New England journal of medicine 2011; 365(17):1567-1575.
3. Vestbo J, Hurd SS, Agusti AG, et al. Global strategy for the diagnosis, management, and prevention of chronic obstructive pulmonary disease: GOLD executive summary. American journal of respiratory and critical care medicine 2013; 187(4):347-365.

4. van Beek EJ, Dahmen AM, Stavngaard T, et al. Hyperpolarised $3 \mathrm{He}$ MRI versus HRCT in COPD and normal volunteers: PHIL trial. The European respiratory journal 2009; 34(6):1311-1321.

5. Kirby M, Mathew L, Heydarian M, et al. Chronic obstructive pulmonary disease: quantification of bronchodilator effects by using hyperpolarized (3) He MR imaging. Radiology 2011; 261(1):283-292.

6. Fain SB, Gonzalez-Fernandez G, Peterson ET, et al. Evaluation of structure-function relationships in asthma using multidetector CT and hyperpolarized He-3 MRI. Academic radiology 2008; 15(6):753-762.

7. Svenningsen S, Kirby M, Starr D, et al. What are ventilation defects in asthma? Thorax 2014; 69(1):63-71.

8. Mentore K, Froh DK, de Lange EE, et al. Hyperpolarized HHe 3 MRI of the lung in cystic fibrosis: assessment at baseline and after bronchodilator and airway clearance treatment ${ }^{1}$. Academic radiology 2005; 12(11):1423-1429.

9. Sheikh K, Paulin GA, Svenningsen S, et al. Pulmonary ventilation defects in older never-smokers. J Appl Physiol (1985) 2014; 117(3):297-306.

10. Woodhouse N, Wild JM, Paley MN, et al. Combined helium-3/proton magnetic resonance imaging measurement of ventilated lung volumes in smokers compared to never-smokers. Journal of magnetic resonance imaging : JMRI 2005; 21(4):365-369.

11. Wang C, Mugler JP, 3rd, de Lange EE, et al. Lung injury induced by secondhand smoke exposure detected with hyperpolarized helium-3 diffusion MR. Journal of magnetic resonance imaging : JMRI 2014; 39(1):77-84

12. Fain SB, Panth SR, Evans MD, et al. Early emphysematous changes in asymptomatic smokers: detection with $3 \mathrm{He}$ MR imaging. Radiology 2006; 239(3):875-883.

13. Kirby M, Pike D, Coxson HO, et al. Hyperpolarized $3 \mathrm{He}$ ventilation defects used to predict pulmonary exacerbations in mild to moderate chronic obstructive pulmonary disease. Radiology 2014;140161.

14. Miller MR, Hankinson J, Brusasco V, et al. Standardisation of spirometry. The European respiratory journal 2005; 26(2):319-338.

15. Kirby M, Heydarian M, Svenningsen S, et al. Hyperpolarized 3 He magnetic resonance functional imaging semiautomated segmentation. Academic radiology 2012; 19(2):141-152.

16. Kirby M, Heydarian M, Wheatley A, et al. Evaluating bronchodilator effects in chronic obstructive pulmonary disease using diffusion-weighted hyperpolarized helium-3 magnetic resonance imaging. Journal of applied physiology 2012; 112(4):651-657.

17. Niimi A, Matsumoto $H$, Amitani $R$, et al. Airway wall thickness in asthma assessed by computed tomography: relation to clinical indices. American journal of respiratory and critical care medicine 2000; 162(4): 1518-1523.

18. West JB. Respiratory physiology: the essentials. 8th ed. Lippincott Williams and Wilkins, 2008.

19. Wan ES, Hokanson JE, Murphy JR, et al. Clinical and radiographic predictors of GOLD-unclassified smokers in the COPDGene study. American journal of respiratory and critical care medicine 2011; 184(1):57-63.

20. Coxson HO, Dirksen A, Edwards LD, et al. The presence and progression of emphysema in COPD as determined by CT scanning and biomarker expression: a prospective analysis from the ECLIPSE study. The lancet Respiratory medicine 2013; 1(2):129-136.

21. Yablonskiy DA, Sukstanskii AL, Woods JC, et al. Quantification of lung microstructure with hyperpolarized $3 \mathrm{He}$ diffusion MRI. Journal of applied physiology 2009; 107(4):1258-1265.

22. Evans A, McCormack D, Ouriadov A, et al. Anatomical distribution of $3 \mathrm{He}$ apparent diffusion coefficients in severe chronic obstructive pulmonary disease. Journal of magnetic resonance imaging: JMRI 2007; 26(6): 1537-1547.

23. Miller RR, Muller NL, Vedal S, et al. Limitations of computed tomography in the assessment of emphysema. The American review of respiratory disease 1989; 139(4):980-983.

24. Coxson HO, Leipsic J, Parraga G, et al. Using pulmonary imaging to move chronic obstructive pulmonary disease beyond FEV1. American journal of respiratory and critical care medicine 2014; 190(2):135-144.

25. Yahaba M, Kawata N, lesato K, et al. The effects of emphysema on airway disease: correlations between multi-detector CT and pulmonary function tests in smokers. European journal of radiology 2014; 83(6):1022-1028.

26. Nakano Y, Muro S, Sakai H, et al. Computed tomographic measurements of airway dimensions and emphysema in smokers. Correlation with lung 
function. American journal of respiratory and critical care medicine 2000; 162(3 Pt 1):1102-1108.

27. Coxson HO. Quantitative chest tomography in COPD research: chairman's summary. Proceedings of the American Thoracic Society 2008; 5(9):874-877.

28. Coxson HO. Quantitative computed tomography assessment of airway wall dimensions: current status and potential applications for phenotyping chronic obstructive pulmonary disease. Proceedings of the American Thoracic Society 2008; 5(9):940-945.
29. Swift AJ, Wild JM, Fichele S, et al. Emphysematous changes and normal variation in smokers and COPD patients using diffusion $3 \mathrm{He}$ MRI. European journal of radiology 2005; 54(3):352-358.

30. Fain SB, Altes TA, Panth SR, et al. Detection of age-dependent changes in healthy adult lungs with diffusion-weighted $3 \mathrm{He} \mathrm{MRI}$. Academic radiology 2005; 12(11):1385-1393.

31. Galban CJ, Han MK, Boes JL, et al. Computed tomography-based biomarker provides unique signature for diagnosis of COPD phenotypes and disease progression. Nature medicine 2012; 18(11):1711-1715. 